\title{
NEVOD as a test facility for future neutrino telescopes
}

\author{
A.A. Petrukhin ${ }^{*}$ and S.S. Khokhlov \\ National Research Nuclear University MEPhI (Moscow Engineering Physics Institute), 115409 \\ Moscow, Russia
}

\begin{abstract}
The possibility to calibrate the new optical modules mDOM of the IceCube-Upgrade neutrino telescope inside the tank of Cherenkov water detector NEVOD is discussed. Methods to calibrate optical modules are presented. The spatial lattice of the detector NEVOD and deployed outside of the water tank calibration telescope system and coordinatetracking detector DECOR allow calibrating the response of $\mathrm{mDOM}$ with respect to the Cherenkov light from muons, muon bundles and cascades with known trajectories.
\end{abstract}

\section{Introduction}

The basic characteristics of water - a large angle of Cherenkov light emission and good transparency for light propagation - make it possible to construct neutrino telescopes (NT) of very large dimensions with a relatively small number of photomultipliers (PMTs) which are housed in optical modules (OMs). One of the important tasks for NTs is the separation of single VHE muons ( $>100 \mathrm{TeV})$, muon bundles and cascades.

An optimal operation of a NT would call for isotropic module sensitivity over a $4 \pi$ solid angle, therefore such a module must consist of several differently oriented PMTs. The idea of a quasi-spherical module was proposed for the first time in 1979 at the 16th International Cosmic Rays Conference (ICRC) [1]. Later it was realized as a quasi-spherical module with six PMTs, the basic element of the Cherenkov water detector NEVOD [2].

First generation underwater neutrino detectors made no (or nearly no) use of the idea of quasi-spherical modules: the IceCube OM houses just one PMT, NT200 in Lake Baikal operated two equally-oriented OMs in coincidence, each with one PMT, and ANTARES has three single-PMT OMs which cover the full azimuth but look essentially downward. Two new projects, however, make already use of modules with a quasi-spherical response: the KM3NeT module contains 31 PMTs, and the baseline OM version for IceCubeUpgrade includes 24 PMTs.

Since the length of cascades in water is only about $10 \mathrm{~m}$, the mapping of their light in NTs with OM spacing larger than $10 \mathrm{~m}$ is difficult.

For the calibration of OMs, usually LED or laser sources with fixed wavelengths are used. They cannot imitate the continuous spectrum and the directionality of the Cherenkov radiation sufficiently well. Therefore the calibration of these OMs in a Cherenkov water

* Corresponding author: AAPetrukhin@mephi.ru 
detector with a close spacing of OMs $(\sim 2 \mathrm{~m})$ is advantageous. NEVOD is presently worldwide the only setup which meets these requirements.

\section{Experimental complex NEVOD}

The complex is located in the campus of the National Research Nuclear University MEPhI in a special four-story building. The basis of the complex is the Cherenkov water detector NEVOD with a tank volume $9 \times 9 \times 26 \mathrm{~m}^{3}$. The detecting system is formed by a spatial lattice of quasi-spherical modules (QSMs), each of them including six PMTs with flat cathodes oriented along the coordinate axes. The QSM allow detecting Cherenkov radiation from any direction with practically the same efficiency. The lattice of QSMs is formed by a set of vertical strings containing three or four modules each. The spacing between the OMs is $2.5 \mathrm{~m}$ and $2.0 \mathrm{~m}$ along the horizontal detector axes, and $2.0 \mathrm{~m}$ in vertical direction. The current detecting system consists of 91 QSMs (546 PMTs in total) [3, 4].

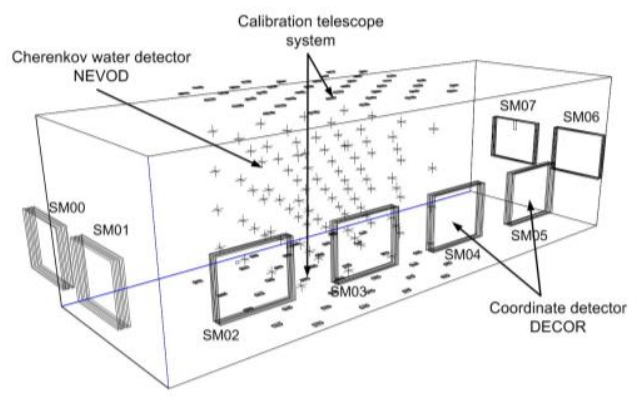

Figure 1. Experimental complex NEVOD.

To improve the event reconstruction accuracy, the coordinate detector DECOR [5] was constructed around the Cherenkov water calorimeter NEVOD (see Figure 1). DECOR represents a modular multi-layer system of plastic streamer tube chambers with resistive cathode coating. It includes eight vertically suspended eight-layer assemblies (supermodules, SMs) of chambers with a total sensitive area $70 \mathrm{~m}^{2}$. The chamber planes are equipped with a two-coordinate external strip readout system that allows to localize charged particle tracks with about $1 \mathrm{~cm}$ accuracy in both coordinates $(\mathrm{X}, \mathrm{Y})$. The directional reconstruction accuracy for muon tracks crossing a single SM is better than $0.7^{\circ}$ (zenith) and $0.8^{\circ}$ (azimuth).

Another part of NEVOD is the calibration telescope system (CTS) [6]. It consists of 2 planes by 40 scintillation counter sandwiching NEVOD as shown in Fig. 1. In both planes, the counters are arranged in a chess board pattern over an area of $8 \times 10 \mathrm{~m}^{2}$. The distance between bottom and top plane is $9.45 \mathrm{~m}$.

\section{Methods to calibrate optical modules with NEVOD}

We have developed two methods to calibrate test optical modules in NEVOD. In the first one, the dependence of the module response on the arrival angle of the Cherenkov radiation is investigated (sphericity test). In the second test, the response of the module on cascade events is studied.

Events with single muons passing through NEVOD were used to study the tested OM response. Such events can be selected using CTS or DECOR. To study the single QSM's ability of determining the direction of Cherenkov light, we selected events, in which the track of the single particle was detected with the calibration telescope system. The selection 
criterion was an activation of one counter in the upper CTS plane and one counter in the lower plane. An example of such an event is shown in Fig. 2 (left). The range of distances from the muon track to the QSM is 0.5 to $5 \mathrm{~m}$.
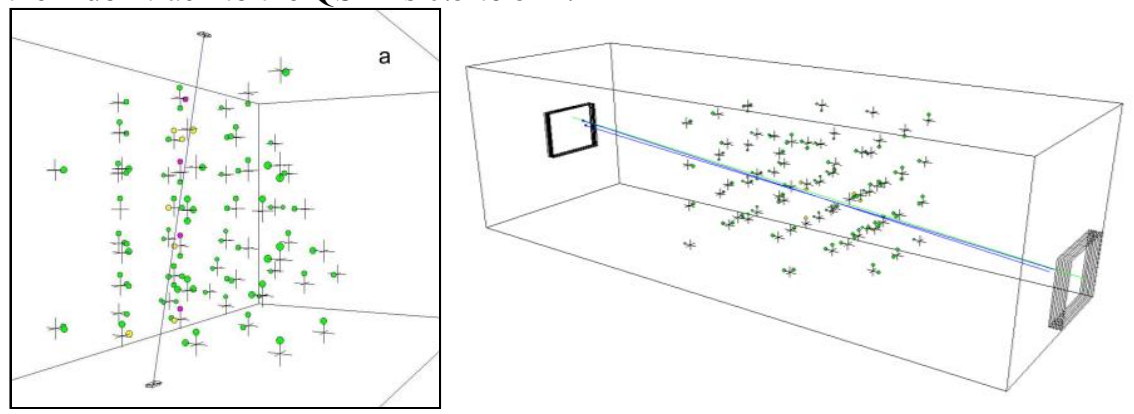

Figure 2. Example of the event detected with CTS (left) and DECOR (right).

The homogeneity of water and the high density of the QSM lattice allow reconstructing the cascade curve of the shower produced by a muon in the sensitive volume of the detector. The technique of reconstruction [7] is based on the amplitude responses of PMTs, which detect the direct light from the cascade core. It is assumed that the electrons and positrons produced in the shower move along the cascade axis and that the axis coincides with the muon track which can be defined with a high precision using DECOR (Fig. 2, right).

So, the problem is to reconstruct the values of two parameters of the theoretical cascade curve in a one-dimensional approximation: the energy of the cascade $\mathrm{E}_{0}$ and the depth on the axis where the cascade was generated. An example of a reconstructed cascade curve of a shower with energy of $860 \mathrm{TeV}$ is shown in Fig. 3.

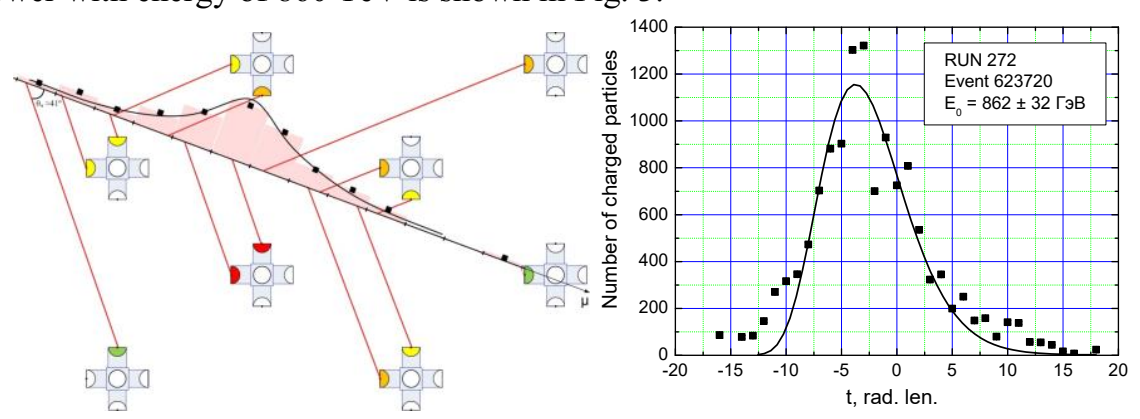

Figure 3. Reconstructed cascade curve of a shower detected in the NEVOD water detector.

\section{Calibration of quasi-spherical module}

A study of the independence of the QSM response from the direction of Cherenkov light (the characteristics of sphericity) was conducted in [8] using events with single muons, detected with a pair of supermodules of DECOR. Two variants of calculation of the QSM response were compared: $B_{1}$ as a sum of amplitudes of PMTs $A_{\mathrm{i}}$, and $B_{2}$ as a square root of the sum of squared PMT amplitudes:

$$
B_{1}=\frac{R}{R_{0}} \sum_{\mathrm{i}} A_{\mathrm{i}}, \quad B_{2}=\frac{R}{R_{0}} \sqrt{\sum_{\mathrm{i}} A_{\mathrm{i}}^{2}}
$$

where $R$ is the distance between muon track and the center of QSM, $R_{0}$ is the normalization distance. It was shown that the response $B_{2}$ has a more narrow distribution. 
In each event, the QSMs register the Cherenkov radiation from a single particle. Its track was defined with DECOR. For the QSM with six PMTs, normals to PMTs divide the sphere into eight equal spherical octants (Figure 4, left). Each of these octants may be divided into six similar spherical triangles, covering the whole sphere using rotations and mirror reflections relative to the symmetry planes of the QSM (Figure 4, right). The parameters of the response of each QSM were projected to theise spherical triangle.

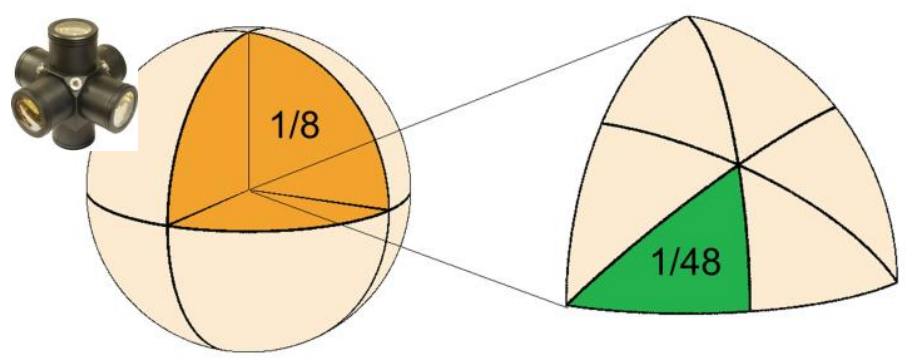

Figure 4. Selection of spherical segments for studying QSM response.

The study was conducted for two ranges of distances $R$ between muon track and center of QSM: $0.5-1.0 \mathrm{~m}$ and $1.0-2.0 \mathrm{~m}$, respectively. The middle of each range was used as normalization distance $R_{0}(0.75 \mathrm{~m}$ and $1.5 \mathrm{~m}$, respectively). The zenith angles of single muons were limited to the interval $86^{\circ}-89^{\circ}$. The average values of $B_{2}$ in photoelectrons defined by equation (1) for various locations on the spherical segment are shown in Fig. 5, a (projection to a plane). The borders of the projection of the spherical segment are marked with light lines.

For each of the two ranges, the rms-deviation from the mean value of the QSM response averaged over the sphere was calculated (Fig. 5, b). This value characterizes the deviation from sphericity of a QSM. It is $8 \%$ for the range of $0.5-1.0 \mathrm{~m}$ and less than $5 \%$ for the range of $1.0-2.0 \mathrm{~m}$.
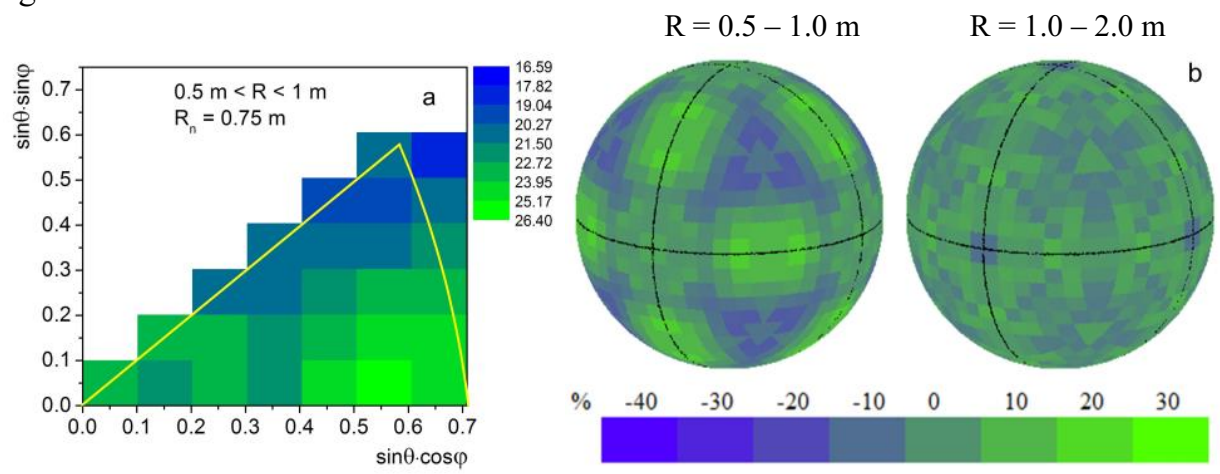

Figure 5. The triangular matrix of QSM response in photoelectrons (a); relative QSM response for two ranges of distances from the center of QSM to the track of the particle (b).

\section{Calibration of mDOM of IceCube-Upgrade in NEVOD}

In preparation of a next generation of IceCube, the new optical module $\mathrm{mDOM}$ has been developed [9]. The mDOM consists of 24 PMTs, and the response of such a module should have a good sphericity. To check this, the IceCube group in Muenster proposed to test the $\mathrm{mDOM}$ in NEVOD. 
Two variants of mDOM calibration in NEVOD have been developed. The mDOM can be placed somewhere between NEVOD's QMs, or it can be integrated in the spatial lattice of NEVOD, replacing one QSM (Figure 6). In the first case the sphericity of the $\mathrm{mDOM}$ can be measured over a wide range of distances between the module and single muons reconstructed with DECOR. In the second case, the effect of the shadows of neighbored QSMs must be taken into account, but we can measure the response of the mDOM on cascades reconstructed by means of the NEVOD spatial lattice, and also the calibration telescope system will be used for reconstruction of near-vertical muons.

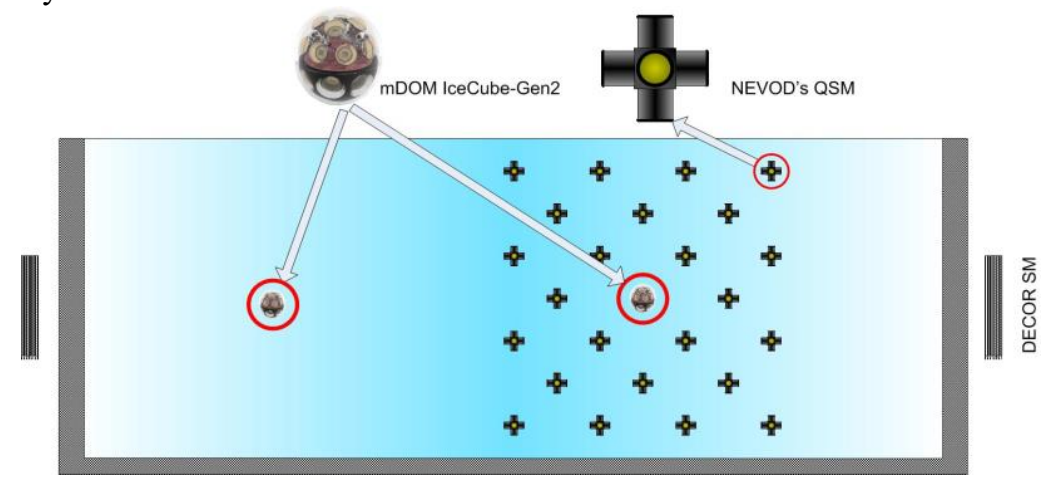

Figure 6. Variants of mDOM calibration in NEVOD.

\section{Conclusion}

Calibration of optical modules of various systems in the volume of the Cherenkov water detector NEVOD using Cherenkov radiation from single muons, muon bundles and cascade showers in unified conditions can help comparing the performance of different neutrino telescopes like IceCube-Upgrade, KM3NeT and Baikal-GVD.

\section{Acknowledgments}

The work was performed at the Unique Scientific Facility "Experimental complex NEVOD" with the financial support provided by the Russian Ministry of Science and Higher Education (state task and MEPhI Academic Excelence Project No. 02.a03.21.0005).

\section{References}

1. V.V. Borog et al., Proc. 16th ICRC, Kyoto, vol. 10, 380 (1979)

2. A.A. Petrukhin, Physics-Uspekhi 58, 486 (2015)

3. V.V. Kindin et al., Physics Procedia 74, 435 (2015)

4. V.V. Kindin et al., Instrum. Exp. Tech. 61, 649 (2018)

5. N.S. Barbashina et al., Instrum. Exp. Tech. 43, 743 (2000)

6. M.B. Amelchakov et al., Instrum. Exp. Tech. 61, 673 (2018)

7. S.S. Khokhlov et al., Bull. Russ. Acad. Sci. Phys. 77, 638 (2013)

8. V.A. Khomyakov et al., Phys. Procedia 74, 442 (2015)

9. L. Classen and A. Kappes for the IceCube Collaboration, "The multi-PMT optical module for the IceCube-Upgrade", VLVNT Workshop, Dubna, 2-4 Oct. 2018 (these proceedings) 